\title{
The shared reward dilemma on structured populations
}

\author{
Raúl Jiménez • José A. Cuesta • Haydée Lugo • \\ Angel Sánchez
}

\begin{abstract}
In this paper we study the recently introduced "shared reward dilemma" (Cuesta et al. in J Theor Biol 251:253 263, 2008) in the presence of a structure governing the interactions among the population. The shared reward dilemma arises when the prisoner's dilemma is supplemented with a second stage in which a fixed reward is equally distributed among all cooperators. We first extend our previous results on the equilibrium structure of this game to the case of a one-shot game taking place on
\end{abstract}

\footnotetext{
This work was supported by Ministerio de Educación y Ciencia (Spain) under grants ECO2008-05080, SEJ2007-67135 and MOSAICO, and by Comunidad de Madrid (Spain) under grants MOSSNOHO-CM and SIMUMAT-CM.
}

R. Jiménez

Departamento de Estadística, Universidad Carlos III de Madrid, Getafe, Spain e-mail: raul.jimenez@uc3m.es

\section{J. A. Cuesta · A. Sánchez (凶)}

Grupo Interdisciplinar de Sistemas Complejos (GISC), Departamento de Matemáticas, Universidad Carlos III de Madrid, Leganés, Spain

e-mail: anxo@math.uc3m.es

\section{J. A. Cuesta}

e-mail: cuesta@math.uc3m.es

H. Lugo

Departamento de Economía, Universidad Carlos III de Madrid, Getafe, Spain e-mail: hlugo@eco.uc3m.es

\section{A. Sánchez}

Instituto de Ciencias Matemáticas CSIC-UAM-UC3M-UCM, Madrid, Spain

\section{A. Sánchez}

Instituto de Biocomputación y Física de Sistemas Complejos (BIFI),

Universidad de Zaragoza, Zaragoza, Spain 
a regular network. Subsequently, we consider an evolutionary version of the game on both lattices and random networks. We show that the evolutionary game on graphs exhibits important differences with the case of well-mixed populations. In particular, there exists an important parameter range in which the cooperation is boosted and a single cooperator can invade a population of defectors. We study the dependence of the cooperation levels on the neighborhood size, finding that on random networks the level of cooperation reached decreases as the neighborhood size increases. Moreover, square lattices favor cooperation more than random networks, and on them cooperation may be almost full for certain parameter regions even for large neighborhood sizes. Further, we show that the effect of the population structure is never detrimental for cooperation. We interpret our results in terms of weak versus strong temptation and discuss the nontrivial issues involved in trying to promote cooperation exogenously by means of such a reward mechanism.

Keywords Emergence of cooperation - Prisoner's dilemma - Shared reward dilemma $\cdot$ Structured population $\cdot$ Evolutionary games

\section{Introduction}

The last decade has witnessed a remarkable increase of the effort devoted to understanding the emergence of cooperation both in animal and human societies (Hammerstein 2003), in spite of which the issue remains largely unsolved (Pennisi 2005). The natural framework for this research is the study of social dilemmas (Kollock 1998), in other words, the study of the tension between individual and collective rationality. In a social dilemma, individually reasonable behavior leads to a situation in which everyone is worse off. Paradigmatic examples of such dilemmas widely applied to the study of cooperative phenomena are the Prisoner's Dilemma and the Snowdrift game (see, e.g., Doebeli and Hauert 2005 and references therein). To date, several mechanisms have been identified that prevent the deleterious effect of competition and natural selection on cooperation: kin selection, direct reciprocity, indirect reciprocity, network reciprocity and group selection (see, e.g., Nowak 2006 and references therein). These mechanisms apply in general to the support of a cooperating population in the presence of defectors, i.e, they work by counteracting in some way the evolutive advantage of defectors. However, these are survival phenomena, not promotion phenomena, in the sense that in case there are none or very few cooperators their population will end up composed by defectors alone. Other factors have been proposed to play a role in the emergence of cooperation, such as punishment (Fehr and Gächter 2003), voluntary participation (Hauert et al. 2007) or a degree of freedom in choosing partners (Poncela et al. 2008), to name a few, but these only takes the problem one step backwards in so far as one has yet to explain the origin of these features.

Promotion of cooperation is indeed a socioeconomically relevant question, that certainly deserves further research and to which the present paper intends to contribute. To this end, we study a simple mechanism for the emergence of cooperation, which we introduced in Cuesta et al. (2008) and which we refer to as the shared reward. In this setting, players interact through a standard Prisoner's Dilemma, but in a second stage 
cooperators receive an additional payoff coming from an exogenous source available only to them and not to defectors. While this is clearly a simple model of incentives to cooperation in socio-economic context, it is interesting to note that similar reward mechanisms can be found in a number of specific applications. This is the case, for instance, of mutualistic situations with selection imposed by hosts rewarding cooperation or punishing less cooperative behavior (see, e.g., Kiers et al. 2003 and references therein). Another context where our model is likely to apply is team formation in animal societies (Anderson and Franks 2001), e.g., in cooperative hunting (Packer and Ruttan 1988). On the other hand, the idea of a shared reward could be implemented in practice as a way to promote cooperation in human groups or, alternatively, may arise spontaneously in situations where costly signaling prior to the game is allowed, if the exchange of cooperative signals among cooperators is free (Skyrms 2006). Previous research (Jiménez et al. 2008) has shown that establishing such a shared reward makes it possible for a single cooperator to invade a population of defectors when strategies evolve by unconditional imitation (Nowak and May 1992): Indeed, cooperation persists after the additional resource has been exhausted or turned off. We have also analyzed the behavior of the model on a well-mixed population under replicator dynamics, with similar results (Cuesta et al. 2008).

Our specific purpose in this paper is to extend our previous analysis of the shared reward mechanism (Cuesta et al. 2008) to the case in which the interactions among players are governed by a social network (Wasserman and Faust 1994) and the strategies of agents evolve by comparing their payoffs with those in their neighborhood. Such an endeavour takes us into the realm of evolutionary game theory on graphs, a context within which a great deal of work has been carried out recently (Szabó and Fáth 2007) building on from the pioneering paper by Nowak and May (1992). We will present our results according to the following scheme: In the next section we extend some of our analytical results (Cuesta et al. 2008) about the shared reward dilemma to the case of a regular network, when the game is played oneshot. Subsequently, in Sect. 3 we introduce the evolutionary version of the shared reward dilemma on different types of networks and report on extensive simulation results. Finally, Sect. 4 summarizes our main findings and discusses their implications.

\section{The shared reward dilemma on networks: the one-shot game}

Consider $N$ players occupying the nodes of a network. Each player can choose one out of two actions: cooperate or defect with her neighbors in an one-shot game (i.e., all player's actions are synchronously performed). We assume that there is no isolated node in the network and all players collect payoffs according to a Prisoner's Dilemma from every one of their links. In addition, players who have chosen to cooperate obtain an extra payoff coming from a fixed reward $\rho$, provided by an external source, that is evenly distributed among all cooperators in the network. This exogenous contribution is what we call a shared reward. It is clear that when there are very few cooperators, the contribution from the shared reward to the total payoff may be sizeable, its importance decreasing as the number of cooperators increases. Hence, 
by introducing this shared reward we are modifying the dilemma arising from the Prisoner's Dilemma, changing it into another one that arises because the reward makes cooperation favorable only if few players cooperate (not unrelated to the so-called Minority game introduced in Challet and Zhang (1997)).

To provide the strategic form of this game we introduce some notation. Let $K$ be the total number of cooperators in the network, and $k_{i}$ the total number of cooperators out of the $n_{i}$ neighbors of player $i$. Payoffs of pairwise interactions are denoted by the standard parameters of the Prisoner's Dilemma: a defector that exploits a cooperator obtains the temptation $T$, but when she faces up another defector she receives the punishment $P$; instead, the payoff for a cooperator meeting another cooperator is the reward $R$ (not to be confused with $\rho$, the reward to be shared that we propose in this work), but obtains the sucker's payoff $S$ when she confronts a defector. For the game to be a Prisoner's Dilemma, payoffs must be ordered according to $T>R>P>S$. The total payoff of player $i$ when the shared reward is taken into account is given by

$$
U_{i}= \begin{cases}k_{i} R+\left(n_{i}-k_{i}\right) S+\frac{\rho}{K}, & \text { if she cooperates } \\ k_{i} T+\left(n_{i}-k_{i}\right) P, & \text { if she defects. }\end{cases}
$$

Note that the condition for full cooperation on the network to be a Nash equilibrium is

$$
n_{i} R+\frac{\rho}{N} \geq n_{i} T, \quad \text { for all } 1 \leq i \leq N
$$

Similarly, the condition for full defection on the network to be a Nash equilibrium is

$$
n_{i} P \geq n_{i} S+\rho, \quad \text { for all } 1 \leq i \leq N
$$

Following the same parametrization scheme of Cuesta et al. (2008), we introduce the parameters

$$
\delta=\frac{\rho}{(T-R) \sum_{i=1}^{N} n_{i}} \quad \text { and } \quad \zeta=\frac{T-R}{P-S}
$$

which will henceforth be referred to as scaled reward and defection ratio respectively. It turns out that for regular networks the analysis of the game can be based in terms solely of these two parameters instead of the five parameters that originally define the game.

For regular networks (i.e. if $n_{i}=n$ for all $1 \leq i \leq N$ ), the conditions for full cooperation and full defection to be Nash equilibria become, respectively, $\delta \geq 1$ and $\delta \leq 1 / N \zeta$. Moreover, the proof of Theorem 1 in Cuesta et al. (2008) can be adapted to show that for any regular network with degree $n \geq 3$, the number of symmetric Nash equilibria in mixed strategies depends only on the values of $\delta$ and $\zeta$. Specifically, the changes in the structure of equilibria when $\delta$ increases are described by one out of 

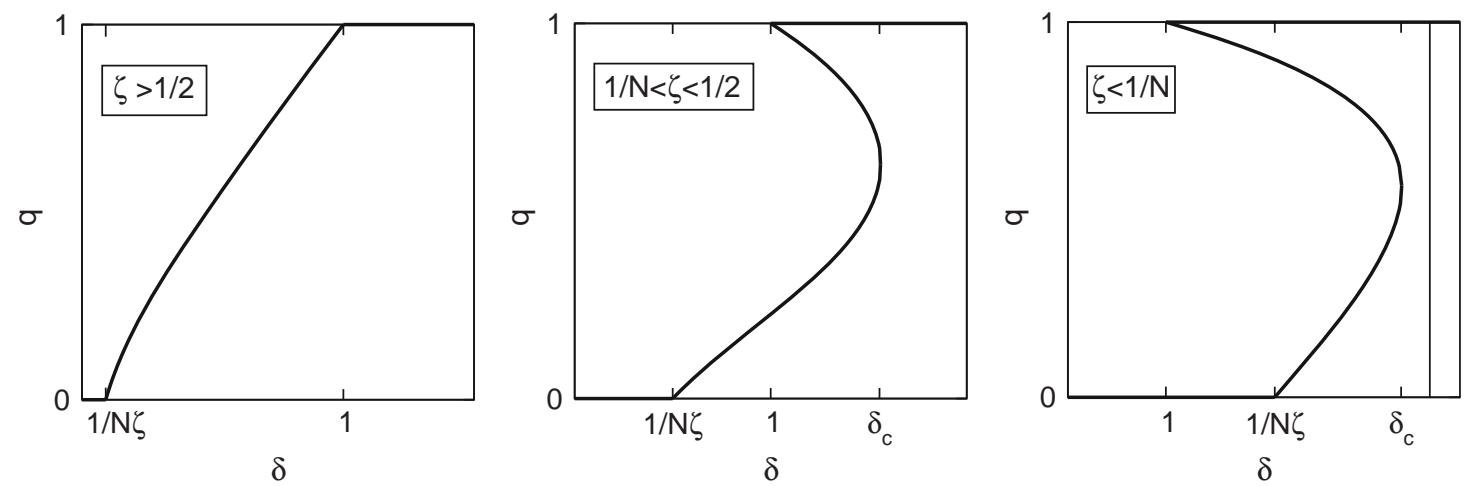

Fig. 1 The three possible scenarios for Nash equilibria in mixed strategies, given in terms of the expected fraction of cooperators, $q$, of the one-shot shared reward dilemma on regular networks with degree greater than or equal to 3 . Corresponding $\zeta$ values are indicated in the plots

three possible scenarios, determined by $\zeta<1 / N, 1 / N \leq \zeta<1 / 2$ and $\zeta \geq 1 / 2$. Figure 1 depicts the typical structure of equilibria for these three cases.

For the case $\zeta \geq 1 / 2$, we can show that there exists a unique symmetric Nash equilibrium which is a continuous increasing function of $\delta$. It is strictly increasing within $[1 / N \zeta, 1]$ from full defection at $\delta=1 / N \zeta$ to full cooperation at $\delta=1$, and constant outside that interval. However, when $\zeta<1 / 2$ we have two nontrivial, different scenarios. One feature common to both of them is the existence of a range of rewards, namely $\max \{1,1 / N \zeta\}<\delta<\delta_{c}$, for which two symmetric equilibria in mixed strategies coexist. One of these equilibria increases and the other decreases when the reward increases within this range. At the critical value $\delta_{c}$ these equilibria collapse and a further increase in $\delta$ yields a discontinuous jump from a Nash equilibrium with $q<1$, $q$ being the expected fraction of cooperators in the network, to full cooperation. The fundamental difference between the cases $\zeta<1 / N$ and $1 / N<\zeta<1 / 2$ arises in the region $\min \{1,1 / N \zeta\}<\delta<\max \{1,1 / N \zeta\}$, where there exists a unique equilibrium with $0<q<1$ : for $1 / N<\zeta<1 / 2$ we see that $q$ increases with $\delta$, while for $\zeta<1 / N$, we see that $q$ decreases with $\delta$. We note that this last case, $\zeta<1 / N$, is somewhat artificial in the case of lattices or networks, where $N$ is general quite large, and therefore we will not consider it.

The next question would naturally be whether these results carry over to nonregular networks. In fact, one can see that generalizing the above results to that case is far from trivial: Indeed, the symmetric mixed equilibria discussed above might not exist at all in a situation in which the players know which node are occupying and how many opponents they are playing with. One can then imagine specific examples of neighborhoods in which being a cooperator or a defector becomes the best option. Therefore, we have not been able to derive analytical results for that case, not even for simple well-known networks such as the Erdös Rènyi one. We will consider those choices in the evolutionary version of the game which we discuss below.

\section{The shared reward dilemma on networks: the evolutionary game}

Following our study of the one-shot game on networks, we extended our research to the evolutionary case. For our analysis, we resorted to agent-based simulations, 
as it is usual in the literature, e.g. Hauert (2006). One question we were particularly interested in was the influence of the size of the neighborhood considered in the levels of cooperation reached in the long run. On the other hand, as stated in the introduction, we were interested in mechanisms that promote cooperation and not only allow for its survival, and hence we studied the invasion of a population consisting only of defectors by a single cooperator. We chose to focus on particular examples of the following scenarios for regular networks with large number of agents:

1. Parametric settings with a unique mixed equilibrium, which correspond to $\zeta>1 / 2$, or $\zeta<1 / 2$ with $\delta<1$.

2. Parametric settings with two mixed equilibria, which correspond to $\zeta<1 / 2$ and $1<\delta<\delta_{c}$.

With the above ideas in mind, we carried out our simulations as follows: For every choice of parameters we considered a single cooperator among a population of defectors. The total number of agents was 2,025 ( $45 \times 45$ in the case of square lattices). Other simulations with different numbers of agents between $N=1,000$ and $N=10,000$ give the same qualitative results. Evolutionary dynamics was implemented by means of the proportional update rule (Szabó and Fáth 2007): at every time step every agent chose one of her neighbors at random. In case this agent had a better payoff than she, then she copied the neighbor's strategy; otherwise, the agent was left unchanged. This is a speeded-up version of the original rule, in which the change of strategy was decided with a probability proportional to the payoff difference; we chose to set this probability to one for any positive difference to cut down on simulation times and because we were interested in stationary states and not in transients (in any event, we checked that choosing a probability proportional to the payoff difference did not change the results). In this respect, it is important to make it clear that the only truly stationary states in our agent based model are full cooperation and full defection, i.e., the absorbing states. We will nevertheless follow the general practice to refer to very long-lived states as stationary. Note that the update of agents' strategy takes place synchronously, i.e., at a given time step all agents update their decision simultaneously. The process is repeated and the fraction of cooperating agents $q$ is monitored. The simulation is run for a 150 time steps and then $q$ is computed by averaging over last 50 time steps. This is subsequently repeated until the values of $q$ obtained in two successive periods agree to two decimal places. At this moment the simulation is stopped and the so obtained value of $q$ is stored. Finally, for every choice of parameters, 100 realizations were simulated according to this procedure. The values of $q$ reported below correspond to the average over realizations.

Simulations are carried out on square lattices with periodic boundary conditions and Erdös Rènyi random networks (Erdös and Rényi 1959) without isolated nodes. In order to compare the results obtained from the different types of networks in the same plot, we define the neighborhood radius as the square root of the mean degree of the network divided by 2 . For regular lattices, the mean degree is the degree of every node, but for random networks this is clearly not the case, therefore the only choice is to use the mean degree. The neighborhoods considered in the regular lattices were rhombuses and squares, extending the well-known von Neumann and Moore neighborhoods: In particular, when the neighborhood radius is 1 , the rhombuses correspond to the von 

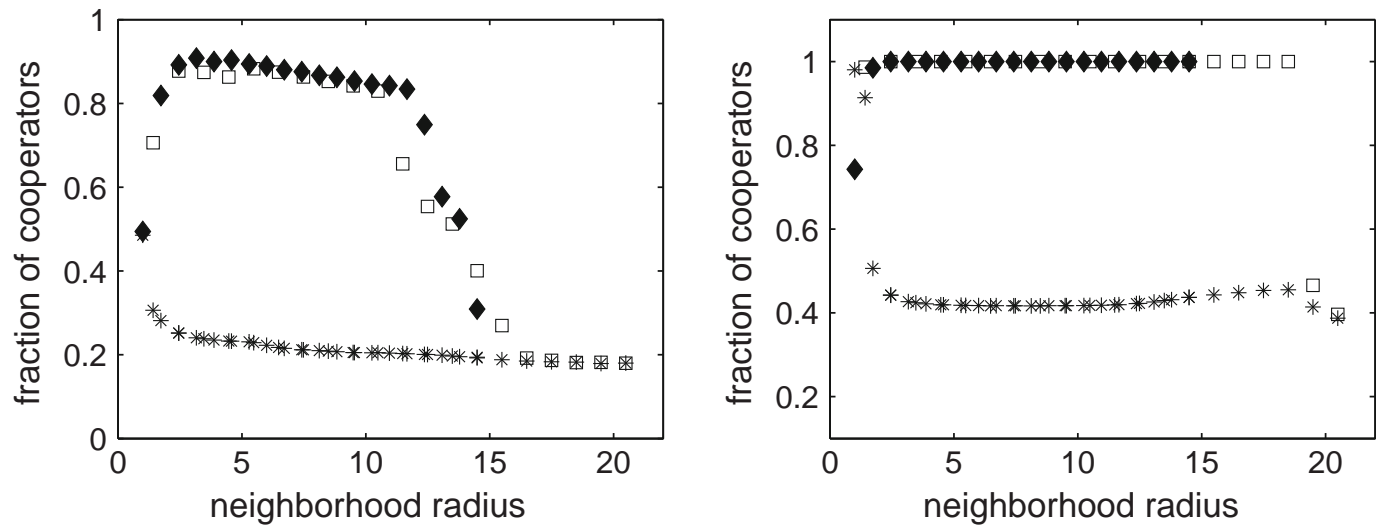

Fig. 2 Fraction of cooperators versus neighborhood radius for $\zeta=0.15$ and $\delta=1.2$ (left) and $\delta=1.8$ (right) (for reference, note that $\delta_{c} \simeq 2$ ). Note that in this case we are in the scenario $\zeta<1 / 2$, with two equilibria in mixed strategies in the one-shot game on lattices. The values of those equilibria correspond, respectively, to $q=0.22$ and $q=0.95$, on one hand, and to $q=0.42$ and $q=0.76$ on the other. Stars correspond to the random Erdös-Rényi network, diamonds to the square lattice with rhombic neighborhoods, and squares to the square neighborhoods

Neumann cells with 4 neighbors. When the neighborhood radius is $\sqrt{2}$ the squares correspond to the Moore cells with 8 neighbors. We note in passing that the effect of different neighborhood sizes has been previously considered in Ifty et al. (2004).

We now report on the results of our simulation program. First, for large $N$, we found that the results depend only on $\zeta, \delta$, and the neighborhood radius. Subsequently, we explored different values of those parameters. The ones we show and discuss in detail below are representative of the features observed in the main cases. Namely,

i) $1 / 2<\zeta$, where we distinguish between $1 / 2<\zeta<1$ and $\zeta>1$. The value of $\delta$ was not qualitatively relevant, unless it is either too small or too large and takes the system very close to the absorbing states.

ii) $\zeta<1 / 2$, where we distinguish between the cases with small or large $\delta \in\left(1, \delta_{c}\right)$.

Figure 2 depicts a typical example of the phenomena observed when $\zeta<1 / 2$. From the discussion in the preceding section, we know that for the one-shot version on the game played on regular lattices there are two equilibria. As the figure shows, our evolutionary dynamics takes the networks, both lattices and random, to a mixed equilibrium stationary state. It is apparent from the figure that for a wide range of neighborhood radius lattices yield much larger cooperation levels than random networks. For small neighborhood radius, we observe that the performance in terms of cooperation of both networks is similar, reaching stationary states with a $50 \%$ of cooperators. This is already a remarkable result in so far as we are simulating invasion by a single cooperator: We thus see that the reward mechanism works, even on lattices and under evolutionary dynamics, to promote cooperation in a population of defectors. The reason why lattices and Erdös Rènyi networks with four neighbors behave similarly is likely to be connected with the fact that the clustering coefficient (the number of triangles in the network divided by the total number of possible triangles) is zero or practically zero in this case and this, as has been recently reported in Roca et al. (2008) makes the behavior of those networks undistinguishable in $2 \times 2$ games. It is then reasonable to believe that a similar mechanism is at work here. In fact, 

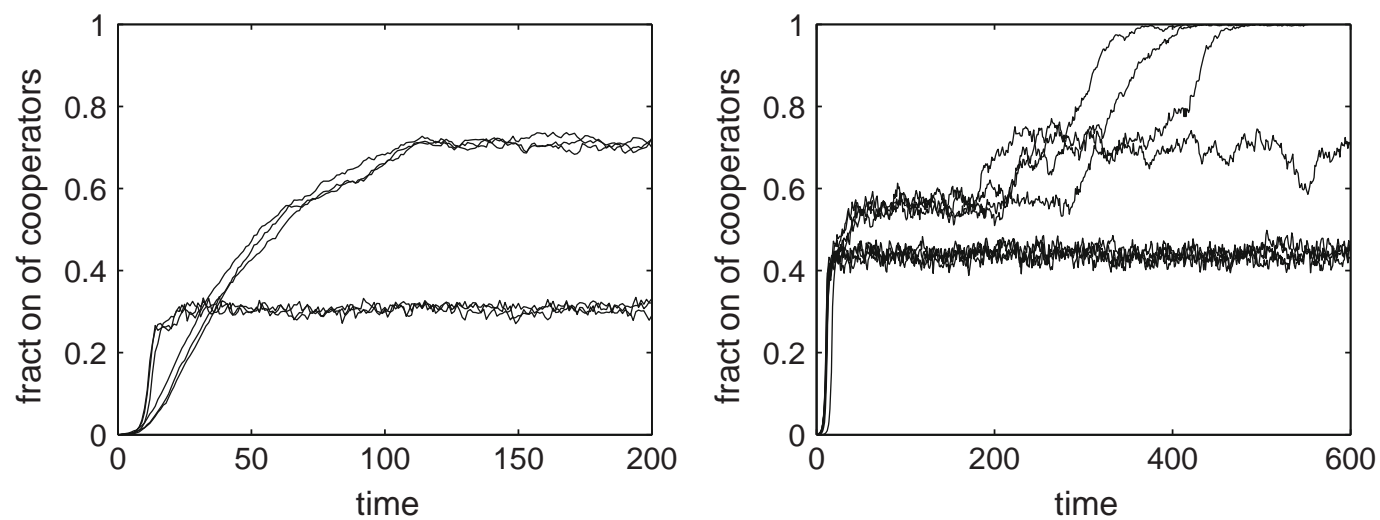

Fig. 3 Density of cooperators versus time for four different realizations for $\zeta=0.15$ and $\delta=1.2$ (left) and $\delta=1.8$ (right) (for reference, note that $\delta_{c} \simeq 2$ ). Left panel: Erdös-Rènyi random networks with average degree 8 and lattices with eight neighbors (Moore neighborhood). The four realizations corresponding to the lattice are those that go to larger values of cooperation. Right panel Erdös-Rènyi random networks with average degrees 8 and 24. The four realizations corresponding to average degree 24 remain in an intermediate cooperation level (slightly above 0.4 ), whereas the remaining four, corresponding to average degree 8 , are trapped in intermediate metastable states before ending up in full cooperation

the role that clustering plays in our model may be connected as well with the observation that, as the neighborhood radius increases, the behavior of lattices and random networks becomes very different. As Fig. 2 shows, on lattices (where clustering is high) the cooperation level increases rapidly with the number of neighbors, until it rapidly reaches a plateau with rather large levels of cooperation. On the contrary, the cooperation level for the random network (where clustering is negligible) decreases initially with the neighborhood radius and subsequently remains constant or almost constant up to the point in which the network becomes the complete graph. This difference between ordered and random networks is more marked in the large $\delta$ case (right panel) than in the small $\delta$ case (left panel), although it is clearly noticeable on both. It is interesting to note that for large $\delta$ lattices reach full cooperation and this survives up to the largest values of the neighborhood radius. Finally, when the neighborhood radius is very large and every node is connected to almost every other one, the details of the structure of the network cease to be relevant and all cases converge to a low value of cooperation, which is close to but different from the one obtained in Sect. 2 for the one-shot case.

In order to understand better the results we have just discussed, Fig. 3 shows several typical realizations seen in our runs for the parameter set of the left panel of Fig. 2. The left panel of Fig. 3 shows the effect of spatial correlations (or clustering) on the improved promotion of cooperation on the square lattice. We observe that Erdös Rènyi networks converge very rapidly to a modest cooperation level, whereas the square lattice takes longer to reach asymptotics as correlations and behavior propagate along the lattice, settling down finally at considerably larger cooperation levels. On the other hand, it is interesting to note that, as shown in the right panel of Fig. 3 when increasing the neighborhood radius in the Erdös Rènyi case, the cooperation level becomes fixed at intermediate levels of cooperation, while realizations with lower number of neighbors have larger average cooperation levels because some realizations are rapidly attracted by the absorbing state at full cooperation. 

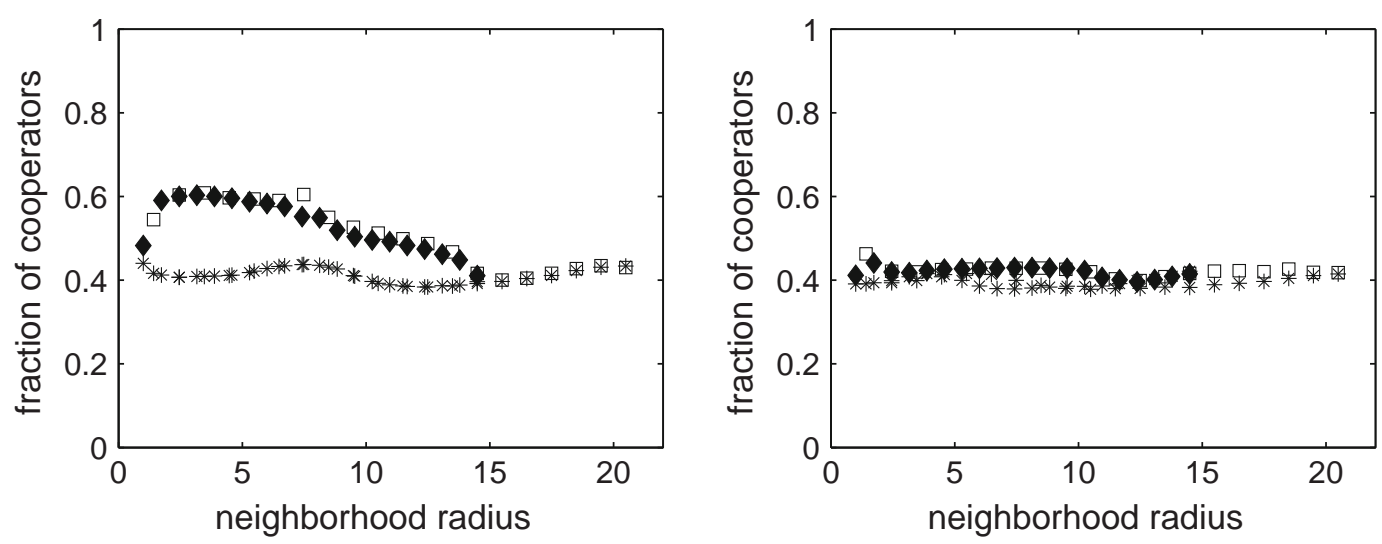

Fig. 4 Asymptotic density of cooperators versus neighborhood radius for $\zeta=0.65$ and $\delta=0.55$ (left panel) and $\zeta=1.15$ and $\delta=0.38$ (right panel). These choices correspond, respectively, to the scenarios $1 / 2<\zeta<1$ and $\zeta>1$, in the region where there is only one equilibrium for the one-shot game on lattices with values $q=0.42$ and $q=0.41$, respectively. Stars correspond to the random Erdös-Rényi network, diamonds to the square lattice with rhombic neighborhoods, and squares to square neighborhoods

The differences between the lattice and the Erdös-Rényi network are much less relevant in the other two possible scenarios, namely $1 / 2<\zeta<1$ and $\zeta>1$ (see Fig. 4). The former exhibits some remnant of the enhanced support for cooperation on lattices when compared to random networks, but is not as dramatic as in the previous case, and the convergence with the cooperation levels attained in the random network takes place for smaller neighborhood radii. As for parameters in the region $\zeta>1$, the right panel of Fig. 4 makes it clear that the results are the same irrespective of working on the ordered or the random network and of the size of the neighborhood. However, it is important to keep in mind that what we are studying here is invasion by a single cooperator, and the resulting cooperation levels are about a $40 \%$ of the population, which is a sizeable fraction. It is also interesting to note that in this cases the convergence for any neighborhood radius is to a value of the cooperation level very close to that found in the one-shot case.

\section{Discussion and conclusion}

As we have established in the previous sections, the first conclusion of the present paper is that the spatial effects in the shared reward dilemma can be very efficient in promoting cooperation in populations consisting entirely of defectors. A single cooperator is enough to initiate an expansion of the cooperative strategy along the network, the resulting values of the fraction of cooperators being dependent on the neighborhood sizes and the specific value of the reward (which, with our parameterization, is contained only in $\delta$ ).

The results of Sect. 2, analytically obtained for regular networks, show a phenomenology which is very similar to that reported for the population without structure in Cuesta et al. (2008). In particular, when $\zeta<1 / 2$, we find coexistence of two mixed equilibria. In this regard, an important unsolved question is the stability of those equilibria for the game on a network. We have not been able to extend our results for the 
well-mixed population to non-regular networks, and thus their equilibrium structure remains an open issue.

The situation is different when we switch to the evolutionary version of the game, where we can consider networks of any kind. Strikingly, the levels of cooperation reached depend on the neighborhood radius and the spatial correlation of the network, when $\zeta<1$. Roughly speaking, the smaller the neighborhood radius is the greater the level of cooperation finally attained. In particular, we observed a remarkable promotion of cooperation on regular lattices when the parameters $\zeta$ and $\delta$ correspond to scenarios with two mixed equilibria. This behavior is opposite to the values of equilibria found for the one-shot game for regular network, which only depend on parameters $\zeta$ and $\delta$. However, for $\zeta>1$, both networks, random and regular ones, reach cooperation levels close to the mixed equilibrium of the one-shot game for regular networks. It is interesting to look deeper into the meaning of the parameter ranges where more cooperation was observed in the evolutionary version. Recalling that $\zeta=(T-R) /(P-S)$, we realize that in this range the temptation to defect, as given by the difference between the temptation payoff and the reward payoff, is smaller than in the other two cases, when $\zeta>1$. We could say that the temptation is then "weak", as it is smaller than the danger of losing by cooperating with a defector. The dramatic promotion of cooperation found in square lattices and the more modest but still significant one obtained in Erdös Rényi networks would then be related to the fact that the reward is able to overcome this "weak" temptation and lead many agents to cooperation. The influence of the reward would then be less noticeable as the temptation moves into a "stronger" regime, as is indeed found in the simulations. We could then conclude that the reward is more efficient when the fear of being exploited is the dominant governing factor and prevails over the temptation to exploit.

Finally, as a more general remark, we stress the fact that we are analyzing a simple mechanism that gives rise to large levels of cooperation in structured populations. Compared to previous studies of games on graphs and claims of sustainment of cooperation (see, e.g., Szabó and Fáth 2007 and references therein), the main difference in our proposal is that the reward does not simply sustain cooperation but it promotes it, in the sense that a single initial cooperator is able to spread the cooperative strategy to a relevant fraction of the population. Of course, as we have already mentioned, even if it is a simple mechanism, it is not at all trivial in so far as larger rewards do not necessarily lead to larger levels of cooperation. To this knowledge, which was already hinted in Cuesta et al. (2008) we can now add the main conclusion of this paper: the effectiveness of the reward depends on the structure of the population and on the size of the neighborhoods, i.e., on the diameter of the set of agents which a given one interacts with. Therefore, application of this proposal in actual situations, specifically, on social networks, would require a careful analysis to foresee the outcome of rewarding cooperation.

\section{References}

Anderson C, Franks NR (2001) Teams in animal societies. Behav Ecol 12:534-540

Challet D, Zhang YC (1997) Emergence of cooperation and organization in an evolutionary game. Phys A 246:407-414 
Cuesta JA, Jiménez R, Lugo H, Sánchez A (2008) The shared reward dilemma. J Theor Biol 251:253-263

Doebeli M, Hauert C (2005) Models of cooperation based on the Prisoner's Dilemma and the Snowdrift game. Ecol Lett 8:748-766

Erdös P, Rényi A (1959) On random graphs, I. Publicationes Mathematicae 6:290-297

Fehr E, Gächter S (2003) The nature of human altruism. Nature 425:785-791

Hammerstein P (ed) (2003) Genetic and cultural evolution of cooperation. MIT Press, Cambridge

Hauert C (2006) Spatial effects in social dilemmas. J Theor Biol 240:627-636

Hauert C, Traulsen A, Brandt H, Nowak MA (2007) Via freedom to coercion: the emergence of costly punishment. Science 316:1905-1907

Ifty M, Killingback T, Doebeli M (2004) Effects of neighbourhood sizes and connectivity on the spatial Continuous Prisoner's Dilemma. J Theor Biol 231:97-106

Jiménez R, Lugo H, Cuesta JA, Sánchez A (2008) Emergence and resilience of cooperation in the spatial Prisoner's Dilemma via a reward mechanism. J Theor Biol 250:475-483

Kiers ET, Rousseau RA, West SA, Denison RF (2003) Host sanctions and the legume-rhizobium mutualism. Nature 425:78-81

Kollock P (1998) Social dilemmas: the Anatomy of Cooperation. Annu Rev Sociol 24:183-214

Nowak MA (2006) Five rules for the evolution of cooperation. Science 314:1560-1563

Nowak MA, May RM (1992) Evolutionary games and spatial chaos. Nature 415:424-426

Packer C, Ruttan L (1988) The evolution of cooperative hunting. Am Nat 132:159-198

Pennisi E (2005) How did cooperative behavior evolve? Science 309:93

Poncela J, Gómez-Gardeñes J, Floría LM, Sánchez A, Moreno Y (2008) Complex cooperative networks from evolutionary preferential attachment. PLoS ONE 3(6):e2449

Roca CP, Cuesta JA, Sánchez A (2008) Sorting out the effect of spatial structure on the emergence of cooperation. http://arxiv.org/abs/0806.1649

Skyrms B (2006) The stag hunt and the evolution of social structure. Cambridge University Press, Cambridge

Szabó G, Fáth G (2007) Evolutionary games on graphs. Phys Rep 446:97-216

Wasserman S, Faust K (1994) Social network analysis: methods and applications. Cambridge University, Cambridge 